\section{Inactivation of Fusidic Acid by Pathogenic Nocardia}

\author{
Ken-Ichi Harada*, KoJi Tomita, Kiyonaga Fuji, \\ Noriko Sato and Hideaki UCHIDA \\ Faculty of Pharmacy, Meijo University, \\ Tempaku, Nagoya 468-8503, Japan
}

\section{Katsukiyo Yazawa and Yuzuru Mikami \\ Research Center for Pathogenic Fungi and \\ Microbial Toxicoses, Chiba University, 1-8-1 Inohana, Chuo-ku, Chiba 260-8673, Japan}

(Received for publication January 13, 1999)

While Nocardia species produce many antibiotics such as $\mathrm{SF} 2475^{1)}$, brasiliquinones ${ }^{2)}$, brasilidine $\mathrm{A}^{3)}$, nocardicyclins ${ }^{4}$, brasilinolide $\mathrm{A}^{5)}$ and brasilicandine $\mathrm{A}^{6)}$, some Nocardia can cause nocardiosis. During studies on the susceptibility of pathogenic Nocardia towards various antibiotics, we have found that pathogenic Nocardia sp. showed species-specific drug resistance patterns ${ }^{7)}$. Our studies also that revealed that most of the resistance in pathogenic Nocardia is due to the inactivation of antibiotics by glycosylation or phosphorylation. Indeed, rifampicin and macrolide antibiotics are inactivated by several Nocardia sp. $^{8 \sim 11)}$.

Fusidic acid (FA, Fig. 1), is a useful antibiotic in the treatment of drug-resistant staphylococcal infections and is produced by fermentation of a strain of the fungus Fusidium coccineum ${ }^{12}$. Chemically, it belongs to a group of tetracyclic triterpenoic acids whose stereochemistry of rings $\mathrm{A}, \mathrm{B}$ and $\mathrm{C}$ is trans-syn-trans, which forces ring $\mathrm{B}$ into a boat conformation. As will be described later, although much research on microbiological transformations of FA has been conducted, we have found a new inactivation product of FA. In the present report we describe the inactivation of FA by a Nocardia brasiliensis to give three products designated as FAIP-1, -2 and -3 , two of which (FAIP-1 and -2) were successfully isolated by chromatography.

The inactivation of FA was carried out and the inactivation products were extracted with ethyl acetate in acidic conditions. Since FA and related compounds were easily detected with a spray reagent composed of vanillin-phosphoric acid, it was possible to differentiate fermentation broth components of $N$. brasiliensis IFM 0329 from FA and related compounds. In a TLC chromatogram two prominent spots (FAIP-1 and 2) were observed together with a faint spot (FAIP-3) and that of FA. The HPLC chromatogram of the same reaction product showed three peaks, FAIP-1 and -2 and FA, but FAIP-3 was not detected. Sephadex LH-20 chromatography is very effective for separation of the FA related compounds from the fermentation broth components of N. brasiliensis.

The structures of FAIP-1 and -2 were spectroscopically determined by the comparison with FA. The FAB mass spectrum of FAIP-1 shows a protonated molecule $(\mathrm{M}+\mathrm{H})^{+}$at $m / z 457$, indicating that FAIP-1 has a molecular weight of 456 . This value corresponds to the loss of acetic acid from FA. The ${ }^{13} \mathrm{C}$ and ${ }^{1} \mathrm{H}$ NMR chemical shifts of FAIP-1 are summarized in Table 1 and are consistent with those of a reported inactive lactone derivative ${ }^{13)}$. Additionally, it was confirmed that

Fig. 1. Structure of fusidic acid (FA).

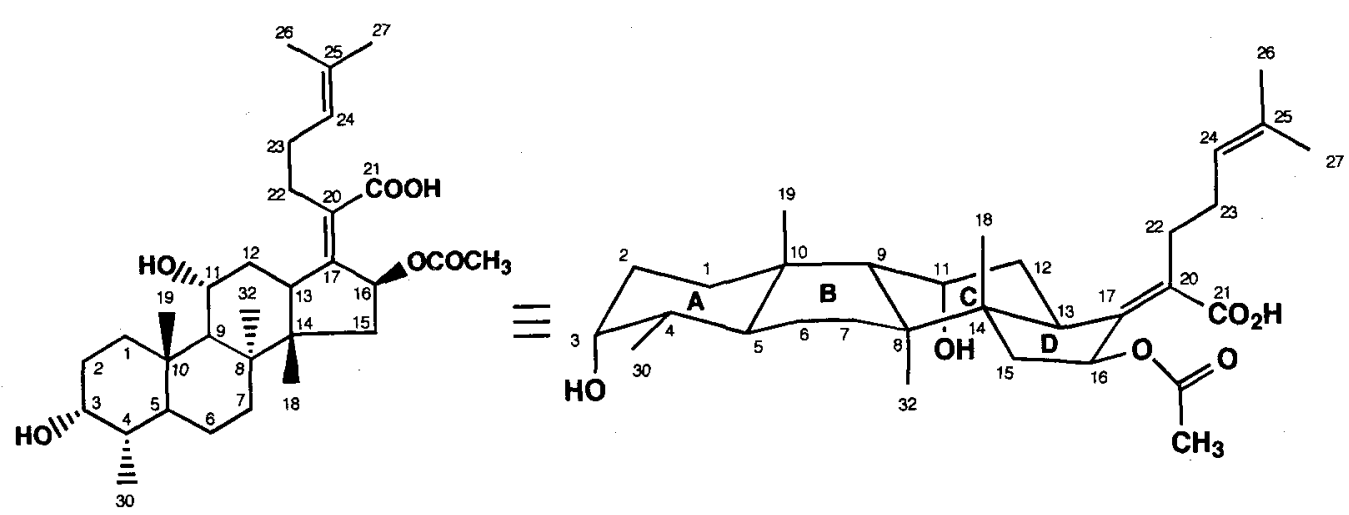


Table $1 .{ }^{13} \mathrm{C}$ and ${ }^{1} \mathrm{H}$ NMR chemical shifts of FA, FAIP-1 and FAIP-2.

\begin{tabular}{|c|c|c|c|c|c|c|c|}
\hline & FA & FAIP-1 & FAIP-2 & & FA & FAIP-1 & FAIP-2 \\
\hline carbon & $\delta_{c}$ & $\delta_{c}$ & $\delta_{c}$ & proton & $\delta_{H}$ & $\delta_{H}$ & $\delta_{H}$ \\
\hline$C-1$ & $\sim 30$ & 29.3 & 29.0 & $1-H$ & $1.35 \sim 1.60,2.05 \sim 2.25$ & $1.28 \mathrm{t}, 2.38 \mathrm{dd}$ & $1.23 \mathrm{t}, 2.43 \mathrm{dd}$ \\
\hline $\mathrm{C}-2$ & $\sim 30$ & 30.1 & 30.0 & $2-H$ & $1.35 \sim 1.60$ & $1.50 \mathrm{~m}, 1.64 \mathrm{~m}$ & $1.49 \mathrm{~m}, 1.61 \mathrm{t}$ \\
\hline $\mathrm{C}-3$ & 69.3 & 69.2 & 68.9 & $3-\mathrm{H}$ & 3.52 & $3.52 \mathrm{~s}$ & $3.47 \mathrm{~s}$ \\
\hline $\mathrm{C}-4$ & 36.2 & 36.7 & 37.2 & $4-H$ & $1.35 \sim 1.55$ & $1.38 \mathrm{~m}$ & $1.31 \mathrm{~m}$ \\
\hline$C-5$ & 35.2 & 34.9 & 35.1 & $5-\mathrm{H}$ & 2.10 & $2.09 \mathrm{~m}$ & $2.17 \mathrm{~m}$ \\
\hline $\mathrm{C}-6$ & 20.7 & 21.0 & 34.1 & $6-\mathrm{H}$ & $0.95 \sim 1.05,1.35 \sim 1.55$ & $1.05 \mathrm{~m}, 1.55 \mathrm{~m}$ & $1.33 \mathrm{~m}, 1.41 \mathrm{~m}$ \\
\hline C-7 & 30.2 & 30.9 & 69.1 & $7-\mathrm{H}$ & $0.95 \sim 1.05,1.35 \sim 1.55$ & $1.05 \mathrm{~m}, 1.55 \mathrm{~m}$ & $3.62 \mathrm{~m}$ \\
\hline $\mathrm{C}-8$ & 39.9 & 40.3 & 45.4 & - & 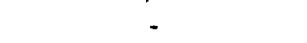 & - & - \\
\hline C-9 & 48.6 & 49.8 & 50.6 & $9-\mathrm{H}$ & 1.33 & $1.33 \mathrm{~s}$ & $1.23 \mathrm{~s}$ \\
\hline C.10 & 36.3 & 36.4 & 36.1 & - & - & - & - \\
\hline C.11 & 66.1 & 65.6 & 65.7 & $11-H$ & 4.13 & $4.19 \mathrm{~s}$ & $4.19 \mathrm{~s}$ \\
\hline C-12 & 36.4 & 31.8 & 31.8 & $12-\mathrm{H}$ & $1.60 \sim 1.70,2.05 \sim 2.25$ & $1.72 \mathrm{dt}, 1.92 \mathrm{dt}$ & $1.68 \mathrm{dt}, 1.91 \mathrm{dt}$ \\
\hline$C-13$ & 41.9 & 37.7 & 37.9 & $13-\mathrm{H}$ & 2.83 & $3.46 \mathrm{~d}$ & $3.46 \mathrm{~m}$ \\
\hline C-14 & 48.2 & 54.8 & 54.4 & $14-H$ & - & - & - \\
\hline C-15 & 38.9 & 33.6 & 37.0 & $15-H$ & $1.09,1.86$ & $1.05 \mathrm{~m}, 2.23 \mathrm{~m}$ & $1.18 \mathrm{dd}, 2.37 \mathrm{~m}$ \\
\hline C-16 & 74.0 & 81.3 & 81.9 & $16-\mathrm{H}$ & 5.75 & $5.12 \mathrm{dd}$ & $5.03 \mathrm{dd}$ \\
\hline C-17 & 139.2 & 171.3 & 171.5 & - & - & - & - \\
\hline C-18 & 17.4 & 19.7 & 18.6 & $18-\mathrm{H}$ & 0.83 & $0.72 \mathrm{~s}$ & $0.75 \mathrm{~s}$ \\
\hline C-19 & 22.7 & 23.3 & 24.0 & $19-\mathrm{H}$ & 0.89 & $0.88 \mathrm{~s}$ & $0.83 \mathrm{~s}$ \\
\hline C-20 & 135.5 & 121.8 & 121.8 & - & - & - & - \\
\hline$C-21$ & 174.9 & 175.7 & 175.9 & - & - & - & - \\
\hline C-22 & $\sim 30$ & 27.0 & 23.4 & $22-\mathrm{H}$ & $2.10 \sim 2.30$ & $2.11 \mathrm{~m}, 2.22 \mathrm{~m}$ & $2.11 \mathrm{~m}, 2.20 \mathrm{dt}$ \\
\hline $\mathrm{C}-23$ & $\sim 30$ & 23.4 & 27.1 & $23-\mathrm{H}$ & $2.10 \sim 2.30$ & $2.11 \mathrm{~m}$ & $2.12 \mathrm{~m}$ \\
\hline$C-24$ & 125.0 & 123.2 & 123.3 & $24-H$ & 5.10 & $5.09 t$ & $5.09 \mathrm{t}$ \\
\hline C-25 & 129.9 & 131.9 & 131.9 & - & - & - & - \\
\hline C-26 & 17.5 & 17.5 & 17.5 & $26-\mathrm{H}$ & 1.57 & $1.56 \mathrm{~s}$ & $1.54 \mathrm{~s}$ \\
\hline $\mathrm{C}-27$ & 25.5 & 25.4 & 25.4 & $27-H$ & 1.64 & $1.65 \mathrm{~s}$ & $1.63 \mathrm{~s}$ \\
\hline $\mathrm{C}-30$ & 16.2 & 16.2 & 16.1 & $30-\mathrm{H}$ & 0.79 & $0.81 \mathrm{~d}$ & $0.78 \mathrm{~d}$ \\
\hline C-32 & 23.5 & 22.3 & 15.1 & $32-\mathrm{H}$ & 1.35 & $1.43 \mathrm{~s}$ & $1.26 \mathrm{~s}$ \\
\hline $\mathrm{CH}_{3}$ & 20.7 & - & - & $\mathrm{CH}_{3}$ & 1.85 & - & - \\
\hline $\mathrm{C}=\mathrm{O}$ & 170.4 & - & - & & & & \\
\hline
\end{tabular}

FAIP-1 is identical with the lactone derivative derived from FA under basic conditions.

The molecular weight of 472 for FAIP-2 was obtained from the $F A B$ mass spectrum, in which the $(\mathrm{M}+\mathrm{H})^{+}$ ion appears at $m / z 473$ and the composition $\mathrm{C}_{29} \mathrm{H}_{45} \mathrm{O}_{5}$ was determined by HRFAB. This molecular weight corresponds to an additional oxygen atom relative to the lactone derivative mentioned above, derivative and FAIP-2 was considered to be a hydroxylated or epoxidised form of FAIP-1 in consideration of the thin layer chromatographic behavior. The ${ }^{13} \mathrm{C}$ and ${ }^{1} \mathrm{H}$ chemical shifts of FAIP-2 are compared with those of FA and FAIP-1 (Table 1), and were assigned using COSY, HMQC and HMBC techniques (Fig. 2 (A)). It was found that the C-7 signal of FAIP-2 is shifted to $\delta$
69.1 and the H-7 signal is observed at $\delta 3.62$ in FAIP-2, indicating that FAIP-2 is 7-hydroxylated FAIP-1. In order to determine the absolute configuration of $\mathrm{C}-7$, the NOESY spectrum was measured. Fig. 2 (B) shows the NOE correlations of FAIP-2 and the NOE correlations are clearly observed between $\mathrm{H}-7 \beta$ and $\mathrm{H}-9$ and $\mathrm{CH}_{3}-19$. This indicates that the hydroxy group is equatorially oriented at C-7, and FAIP-2 is $7 \alpha$-hydroxylated FAIP-1.

Because the molecular weight of FAIP-3 could be deduced to be 488 by the FAB mass spectral analysis, it may be a dihydroxylated derivative of FAIP-1. However, it was not possible to determine its structure due to limited amounts of the isolated compound.

FA is active against the Gram-positive bacteria, Staphylococcus aureus, Micrococcus luteus and Bacillus 
Fig. 2. (A) Structure of FAIP-2 and COSY, HMBC and (B) NOE correlation in FAIP-2.

(A)

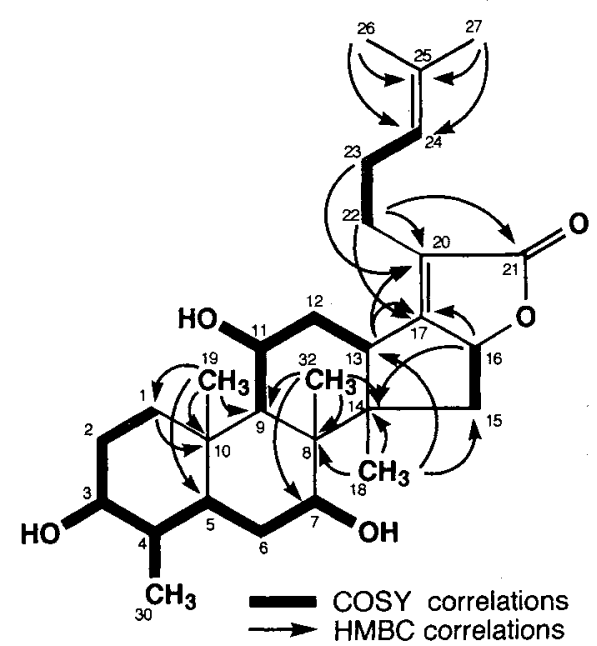

(B)

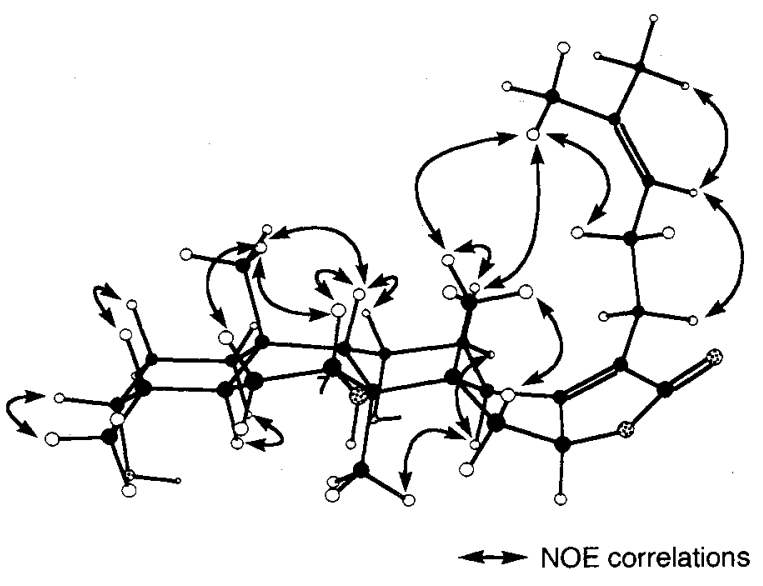

Table 2. Antimicrobial activity of FA, FAIP-1 and FAIP-2.

\begin{tabular}{lccc}
\hline \multirow{2}{*}{ organism } & \multicolumn{3}{c}{ MIC $(\mu \mathrm{g} / \mathrm{ml})$} \\
\cline { 2 - 4 } & FA & FAIP-1 & FAIP-2 \\
\hline Micrococcus luteus IFM 2066 & $\leqq 0.6$ & 5.0 & 660 \\
Bacillus subtilis PCI 189 & $\leqq 0.6$ & 2.5 & $>660$ \\
\hline
\end{tabular}

subtilis $^{14)}$. The antimicrobial activity of FAIP-1 and -2 was examined together with FA using $M$. luteus and Bacillus subtilis as the test organisms (Table 2). As expected, FA showed activity against these two test strains, whereas FAIP-1 showed less activity and FAIP-2 completely lost activity.

FA was discovered in 1962 and since then much research on the inactivation of $\mathrm{FA}$ has been conducted to determine with structure-activity relationships. Modifications of the structure and antibacterial properties of FA were attempted by microbiological processes. A total of 2254 isolates, mainly fungi, streptomycetes and bacteria, were examined and $2.9 \%$ of the organisms were found to inactivate $\mathrm{FA}^{15)}$. Also, a variety of microorganisms including Nocardia sp. which are capable of transforming steroid substrates by means of biooxidative and bioreductive processes were incubated with FA and several conversion products such as 3-keto derivative, 3-epifusidic acid and 16-deacetylfusidic acid were obtained. DAEHNE et $a .^{16)}$ demonstrated that the fungus Acrocylindrium oryzae introduces oxygen functions into positions 6 and 7 of FA and these products showed markedly reduced antibacterial activity. VON DER HAAR et al. $^{13)}$ have demonstrated that several Streptomyces strains are resistant to FA and this resistance is mediated by structural modification of the antibiotic. They obtained two transformed derivatives (having $\mathrm{Rf} 0.61$ and $0.20)$ of FA from the culture broth, and the structure of one $(\operatorname{Rf} 0.61)$ was determined to be a lactone derivative. We have obtained the same compound (FAIP-1) from the culture broth of FA with $N$. brasiliensis IFM 0329 , suggesting that FAIP-2 may correspond to the compound with $\operatorname{Rf} 0.20$. Subsequently, the same authors isolated an extracellular enzyme from Streptomyces lividans, 
which catalyzes the hydrolysis of the acetoxy group at C-16, so that FAIP-1 is formed after spontaneous lactonization ${ }^{17)}$.

We have demonstrated that rifampicin and macrolide antibiotics become inactive against pathogenic Nocardia through the modification of the structures by phosphorylation and/or glycosylation. However, FA becomes inactive through a completely different inactivation process by $N$. brasiliensis IFM 0329, which stereospecifically oxidizes at C-7. Because FAIP-3 may be a further oxidized product of FAIP-2, oxidation is considered to be a key process for the inactivation of FA by $N$. brasiliensis IFM 0329. Although the present study is preliminary, we observed this inactivation of FA with all tested strains of $N$. brasiliensis. This type of inactivation, however, was not observed 'with other species of pathogenic Nocardia, e.g., N. asteroides, N. farcinica, $N$. nova and $N$. otitidiscaviarum. Therefore, the inactivation of FA with oxidation may be a species-specific phenomenon in $N$. brasiliensis.

\section{Experimental}

\section{MIC Determination}

Antimicrobial activities were determined by a microbroth dilution method using brain heart infusion broth (BHI broth, Difco).

\section{Inactivation of Fusidic Acid}

$N$. brasiliensis IFM 0329 (type strain) was inoculated into a $10 \mathrm{ml}$ Erlenmeyer shake flask containing $5 \mathrm{ml}$ of a seed medium ( $2 \%$ glucose supplemented BHI medium). The inoculated flasks were shaken at $250 \mathrm{rpm}$ at $32^{\circ} \mathrm{C}$ for 3 days. The mature seed cultures were inoculated at a $2 \%$ ratio into $100 \mathrm{ml}$ Erlenmeyer flasks containing $50 \mathrm{ml}$ of a medium ( $\mathrm{pH} 7.2$ ) composed of $1 \%$ glucose, $0.05 \%$ $\mathrm{KH}_{2} \mathrm{PO}_{4}, \quad 0.05 \% \quad \mathrm{MgSO}_{4} \cdot 7 \mathrm{H}_{2} \mathrm{O}, 0.4 \%$ sodium glutamate and the culture was incubated at $32^{\circ} \mathrm{C}$ for 1 day. Methanol-sterilized fusidic acid was added at a final concentration of $50 \mu \mathrm{g} / \mathrm{ml}$. The culture was incubated for three more days in the same conditions. The culture was filtered and the $\mathrm{pH}$ of the filtrate was adjusted to 2.0 with $6 \mathrm{M} \mathrm{HCl}$ and the filtrate was extracted with ethyl acetate. After concentration of the extract in vacuo to dryness, the resulting residue was used for the subsequent separation.

\section{Chromatography}

TLC was performed using the following conditions: silica gel plate, Kieselgel $60 \mathrm{~F}_{254}$ (E. Merck, Darmstadt, Germany); mobile phase, benzene: acetone : acetic acid (70:30:1); detection, spraying with vanillin-phosphoric acid, followed by heating at $120^{\circ} \mathrm{C}$ for $10 \sim 20$ minutes. HPLC was carried out using the following conditions: pump, JASCO PU 980 (Tokyo, Japan); detector, JASCO UV-970; column, Cosmosil 5C18-AR $(150 \times 4.6 \mathrm{~mm}$, Nacalai Tesque, Kyoto, Japan); mobile phase, methanol:0.05\% TFA (67:33); detector, UV absorbance at $215 \mathrm{~nm}$; flow rate, $1 \mathrm{ml} /$ minute. Silica gel and Sephadex LH-20 for chromatography were obtained from Fuji silysia (Tokyo, Japan) and Pharmacia (Uppsala, Sweden), respectively.

\section{Purification of Inactivation Products}

The inactivated products mentioned above were subjected to silica gel chromatography using chloroform: methanol $(95: 5 \rightarrow 50: 50)$ as the mobile phase to separate FAIP-1 and FAIP-2 fractions. The FAIP-1 fraction was repeatedly chromatographed on silica gel using benzene : acetone : acetic acid $(90: 10: 1 \rightarrow 70: 30: 1)$ as the mobile phase and the desired fraction was finally purified by Sephadex LH-20 column chromatography using methanol as the mobile phase to give $24.8 \mathrm{mg}$ of FAIP-1. FAIP-1 has the following properties: colorless amorphous powder, $[\alpha]_{\mathrm{D}} 51.4^{\circ}(c 0.58, \mathrm{MeOH})$, positive FABMS $m / z 457(\mathrm{M}+\mathrm{H})^{+}$. The FAIP-2 fraction was repeatedly chromatographed on silica gel using chloroform: methanol $(95: 5 \rightarrow 50: 50)$ and benzene:acetone: acetic acid $(80: 20: 1)$ as the mobile phases and the desired fraction was finally purified by Sephadex LH-20 column chromatography using methanol as the mobile phase to give $3.3 \mathrm{mg}$ of FAIP-2. FAIP-2 has the following properties: colorless amorphous powder, $[\alpha]_{\mathrm{D}} 13.6^{\circ}(c 0.25$, $\mathrm{MeOH})$, positive FABMS $m / z 473(\mathrm{M}+\mathrm{H})^{+}$, positive HRFAB-MS $m / z 473.3260(\mathrm{M}+\mathrm{H})^{+}$calcd. for $\mathrm{C}_{29} \mathrm{H}_{45} \mathrm{O}_{5}$, $\Delta+1.3 \mathrm{ppm}$.

\section{Structure Analysis}

${ }^{1} \mathrm{H}$ and ${ }^{13} \mathrm{C}$ NMR spectra were measured in DMSO- $d_{6}$ on JOEL JNM A-600 and JOEL JNM A-400 spectrometers. Chemical shifts of ${ }^{1} \mathrm{H}$ and ${ }^{13} \mathrm{C}$ were recorded in $\delta$ units relative to the solvent peaks $\left({ }^{1} \mathrm{H}: \delta 2.49\right.$ and ${ }^{13} \mathrm{C}$ : $\delta 39.5$ in DMSO- $d_{6}$ ). FAB mass spectra and HRFAB mass spectra were measured on a JEOL JMX-HX110 spectrometer. Glycerol was used as the matrix.

Conversion of FA to FAIP-1

Sodium fusidate $(50 \mathrm{mg})$ was dissolved in methanol. The solution was adjusted to $\mathrm{pH} 11$ with $5 \% \mathrm{KOH}$ (aq) 
and heated under reflux for 7 hours. The reaction mixture was acidified with $20 \% \mathrm{HCl}$ and the solution was evaporated under reduced pressure. The residual aqueous solution was extracted three times with ethyl acetate. The extract was dried over anhydrous $\mathrm{K}_{2} \mathrm{CO}_{3}$ and evaporated to dryness. The residue was chromatographed on silica gel with benzene : acetone : acetic acid $(90: 10: 1)$ as the mobile phase to give pure lactone derivative $(27.1 \mathrm{mg})$ as an amorphous powder.

\section{Acknowledgment}

This study was partly supported by The Cooperative Research Program of The Research Center for Pathogenic Fungi and Microbial Toxicoses, Chiba University ('97-3).

\section{References}

1) Miyadoh, S.; S. Amano \& S. Takashi: Strain SF2457, a new producer of amicetin group antibiotics. Actinomycetologica 4: $85 \sim 88,1990$

2) Tsuda, M.; H. Sato, Y. Tanaka, K. Yazawa, Y. Mikami, T. Sasaki \& J. Kobayashi: Brasiliquinones $\mathrm{A} \sim \mathrm{C}$, new cytotoxic benz [a] anthraquinones with an ethyl group at C-3 from actinomycete Nocardia brasiliensis. J. Chem. Soc., Perkin Trans. 1: 1773 1775,1996

3) Kobayashi, J.; M. Tsuda, A. Nemoto, Y. Tanaka, K. YazaWA \& Y. MiKami: Brasilidine A, a new cytotoxic isonitrile-containing indole alkaloid from the actinomycete Nocardia brasiliensis. J. Nat. Products 60: $719 \sim 720,1997$

4) Tanaka, Y.; U. Graefe, K. Yazawa, Y. Mikami \& M. RITZAU: Nocardicyclins A and B: new anthracycline antibiotics produced by Nocardia pseudobrasiliensis. J. Antibiotics 50: 822 827, 1997

5) Shigemori, H.; Y. Tanaka, K. Yazawa, Y. Mikami \& J. Kobayashi: Brasilinolide A, new immunosuppressive macrolide from actionomycete, Nocardia brasiliensis. Tetrahedron 52: $9031 \sim 9034,1996$

6) Shigemori, H.; H. Komaki, K. Yazawa, Y. Mikami, A. Nemoto, Y. Tanaka, T. Sasaki, Y. IN, T. Ishida \& J. KobaYASHI: Brasilicandin A, a novel tricyclic metabolite with potent immunosupperssive activity from actinomycete Nocardia brasiliensis. J. Org. Chem. 63: $6900 \sim 6904,1998$

7) Mikami, Y. \& K. Yazawa: Susceptibility patterns of pathogenic Nocardia to some selected antimicrobial agents and their usefulness in the identification works in a clinical laboratory. Bulletin of the Japan Federation for Culture Collections 5: 89 95, 1989

8) Yazawa, K.; Y. Mikami, A. Maeda, M. Akao, N. MORISAKI \& S. IWASAKI: Inactivation of rifampin by Nocardia brasiliensis. Antimicrob. Agents Chemother. 37: $1313 \sim 1317,1993$

9) YaZawa, K.; Y. Mikami, A. Maeda, N. Morisaki \& S. IWASAKI: Phosphorylative inactivation of rifampicin by Nocardia otitidiscaviarum. J. Antimicrob. Chemother. 33: 1127 1135, 1994

10) Yazawa, K.; Y. Mikami, A. Maeda, T. Sakamoto, Y. Ueno, N. Morisaki, S. Iwasaki \& K. Furihata: Inactivation of the macrolide antibiotics erythromycin, midecamycin, and rokitamycin by pathogenic Nocardia species. Antimicrob. Agents Chemother. 38: $2197 \sim 2199,1994$

11) Morisaki, N.; S. Iwasaki, K. Furihata, K. Yazawa \& Y. Mikami: Structural elucidation of rokitamycin, midecamycin and erythromycin metabolites formed by pathogenic Nocardia. Mag. Res. Chem. 33: $481 \sim 489$, 1995

12) von Daehne, W.; S. Jahsen, I. Kirk, R. Larsen \& H. LoRCK: Fusidic acid: properties, biosynthesis, and fermentation. Drugs Pharm. Sci. 22: $427 \sim 449,1984$

13) von der HaAr, B.; D. Rosenberg, W. DitTrich \& H. SCHREMPF: Inactivation of fusidic acid by resistant Streptomyces strains. J. Antibiotics 44: 785 792, 1991

14) Godtfresen, W. O.; W. von Daehne, L. Tybring \& S. VANGEDAL: Fusidic acid derivatives. I. Relationship between structure and antibacterial activity. J. Med. Chem. 9: 15 22, 1966

15) Dvonch, W.; G. Greenspan \& H. E. Alburn: Microbiological oxidation of fusidic acid. Experientia 22: 517,1966

16) VON DAeHne, W.; H. LORCH \& W. O. GodTfRedSEN: Microbiological transformations of fusidane-type antibiotics. A correlation between fusidic acid and helvolic acid. Tetrahedron Lett. 4843 4846, 1968

17) VON DER HAAR, B. \& H. SCHREMPF: Purification and characterization of a novel extracellular Streptomyces lividans 66 enzyme inactivating fusidic acid. J. Bacteriol. 177: 152 155, 1995 